\title{
O impacto do período de pandemia no desempenho ocupacional dos estudantes da área da saúde da Universidade do Estado do Pará - Campus Belém
}

The impact of the pandemic period on the occupational performance of students in the health area of

the University of the State of Pará - Campus Belém

El impacto de la pandemia en el desempeño ocupacional de estudiantes del área de salud de la

\author{
Universidad del Estado de Pará - Campus Belém
}

Recebido: 29/12/2021 | Revisado: 03/01/2022 | Aceito: 10/01/2022| Publicado: 13/01/2022

\author{
Paulo Vitor Santos Da Silva \\ ORCID: https://orcid.org/0000-0003-1571-5626 \\ Universidade do Estado do Pará, Brasil \\ E-mail: Paulo.silvavi30@gmail.com \\ Ana Carolina Gonçalves Rodrigues \\ ORCID: https://orcid.org/0000-0002-6826-3435 \\ Universidade do Estado do Pará, Brasil \\ E-mail: pdqcs@hotmail.com \\ Bianca Rodrigues Da Silva \\ ORCID: https://orcid.org/0000-0002-5949-6827 \\ Universidade do Estado do Pará, Brasil \\ E-mail: bibirsilva@gmail.com \\ Karina Saunders Montenegro \\ ORCID: https://orcid.org/0000-0002-6733-8642 \\ Universidade do Estado do Pará, Brasil \\ E-mail: karinasmonte@yahoo.com.br
}

\section{Resumo}

Em tempos de COVID-19, o distanciamento social é essencial para evitar a propagação do vírus e manter a população protegida, porém, acarreta mudanças na rotina dos indivíduos. Entre estes, pode se destacar os universitários que tiveram mudanças abruptas em suas ocupações. O objetivo desse estudo foi identificar perdas no cotidiano e comprometimentos nas ocupações dos estudantes do Centro de Ciências Biológicas e da Saúde da UEPA. Trata-se de uma abordagem quantitativa, do tipo descritiva e exploratória, de corte transversal, produzida através de um formulário com nove perguntas fechadas e de múltipla escolha, respondida pelos discentes. A maioria dos integrantes da pesquisa eram acadêmicos de Terapia Ocupacional; notou-se que a maior parte dos participantes julga que é extremamente importante ter autocuidado, produtividade e lazer; acerca da realização, a maioria tentou mas não conseguiu realizar como esperado o autocuidado e o lazer, e sobre a produtividade, não conseguiu realizar; além disso, sobre a satisfação em geral foi nada satisfeito, pouco satisfeito e mediano em relação ao lazer, autocuidado e produtividade, respectivamente. Concluiu-se que o distanciamento social acarretou problemáticas no desempenho ocupacional dos discentes participantes, referente às atividades de autocuidado, produtividade e lazer.

Palavras-chave: Desempenho ocupacional; COVID-19; Estudante universitários; Terapia ocupacional.

\begin{abstract}
In times of COVID-19, social distancing is essential to prevent the spread of the virus and protect the population. However, it entails changes in people's routines. Among these are the university students, who needed to have modifications in their daily activities. The objective of this study was to identify losses in daily life and impairments in activities of students of the Center for Biological and Health Sciences of UEPA. This is a quantitative, descriptive and exploratory, cross-sectional approach, produced through a form with nine closed and multiple-choice questions, answered by the students. The majority of the research integrants was Occupational Therapy students. It was noted that most of the participants think that it is extremely important to have self-care, productivity and leisure; regarding the achievement of self-care and leisure, the
\end{abstract}


majority of the participants tried but couldn't do as expected, while no productivity was achieved by them. in addition, regarding satisfaction in achieving leisure, self-care and productivity, the results were mostly "not at all satisfied", "not satisfied" and "kind of satisfied", respectively. In conclusion, the social distancing results in problems on the activities performance of the participant students regarding self-care, productivity and leisure.

Keywords: Occupational performance; COVID-19; College students; Occupational therapy.

\section{Resumen}

En tiempos del COVID-19, la distancia social es fundamental para prevenir la propagación del virus y mantener protegida a la población, sin embargo, conlleva cambios en la rutina de los individuos. Entre estos, los universitarios que tuvieron cambios abruptos en sus ocupaciones. El objetivo de este estudio fue identificar pérdidas en la vida diaria y deficiencias en las ocupaciones de los estudiantes del Centro de Ciencias Biológicas y de la Salud de la UEPA. Es un enfoque cuantitativo, descriptivo y exploratorio, transversal, elaborado a través de un formulario con nueve preguntas cerradas y de opción múltiple, respondidas por los estudiantes. La mayoría de los miembros de la investigación eran académicos de Terapia Ocupacional; Se señaló que la mayoría de los participantes cree que es sumamente importante tener autocuidado, productividad y ocio; sobre el logro, la mayoría lo intentó pero no pudo realizar el autocuidado y el ocio como se esperaba, y en cuanto a la productividad, no pudo desempeñarse; además, sobre la satisfacción en general, no se mostró nada satisfecho, poco satisfecho y medio en relación al ocio, el autocuidado y la productividad, respectivamente. Se concluyó que el distanciamiento social ocasionó problemas en el desempeño ocupacional de los estudiantes participantes, en los cuales, con respecto a las actividades de autocuidado, la productividad y las actividades de ocio.

Palabras clave: Desempeño ocupacional; COVID-19; Estudiantes universitarios; Terapia ocupacional.

\section{Introdução}

Diante dos inúmeros casos de COVID-19 em todo o mundo, a Lei n. 13.979, de 6 de fevereiro de 2020 (Brasil, 2020) dispôs de práticas de enfrentamento da pandemia no país, em especial com a aplicação de quarentena e de distanciamento social em todo território nacional. No dia 18 de março de 2021, o Governo do Estado do Pará suspendeu todas as aulas da rede pública e, também, da Universidade do Estado do Pará (UEPA) (Uepa, 2020).

Durante o tempo decorrido, os estudantes permaneceram em casa, em isolamento, e muitos desses alunos tiveram parte de suas ocupações prejudicadas, devido ao afastamento social e as mudanças no seu cotidiano ocupacional. Juntamente a esse abrupto desligamento de inúmeras atividades cotidianas, há o grande estresse psicológico e emocional acarretado pelo contexto da pandemia em si, e esse conjunto de preocupações, impedimentos, estresses e incertezas afetou diretamente o desempenho ocupacional dos estudantes. Tais fatos desencadearam dificuldades em realizar atividades de autocuidado, produtividade e lazer (Pereira, 2021).

Entende-se como desempenho ocupacional a aptidão que um indivíduo possui para cumprir rotinas e executar papéis e tarefas, com o objetivo de contemplar atividades de autocuidado, produtividade e lazer, atividades essas que são demandas do âmbito externo e interno ao indivíduo (Caldas, 2011).

Esta pesquisa se fundamentou na Medida Canadense de Desempenho Ocupacional (COPM), e abordou o desempenho ocupacional se baseando no modelo canadense de desempenho ocupacional. O COPM abrange as três áreas da ocupação humana (autocuidado, produtividade e lazer) e é voltada para detectar mudanças na autopercepção do indivíduo ao longo do tempo; avaliar o desempenho e a satisfação relacionada à área-problema (Law et al., 2009).

Desse modo, faz-se de grande importância a averiguação e mensuração que o período de isolamento trouxe para o desempenho ocupacional dos estudantes, a fim de que, posteriormente, sejam criadas medidas que possam reverter ou atenuar tal questão.

Os estudos de Acácio et al. (2021) reforçam a necessidade de novos estudos que busquem avaliar o desempenho ocupacional dos individuos durante a pandemia. Assim, foi possível neste trabalho relacionar as ocupações e desempenho ocupacional descritas 
Research, Society and Development, v. 11, n. 1, e49211125229, 2022

(CC BY 4.0) | ISSN 2525-3409 | DOI: http://dx.doi.org/10.33448/rsd-v11i1.25229

no documento de domínio e processo da Terapia Ocupacional com a investigação acerca das atividades cotidianas de estudandes da área da saúde durante a pandemia de covid-19 iniciando em 2020.

Dessa forma, o objetivo desse estudo foi identificar perdas no cotidiano e comprometimentos nas ocupações dos estudantes do Centro de Ciências Biológicas e da Saúde da UEPA. Acredita-se que este estudo irá contribuir para a literatura brasileira, acerca da pandemia de COVID-19.

\section{Metodologia}

O presente estudo requer uma abordagem quantitativa, do tipo descritiva e exploratória, de corte transversal. Utilizou-se registros digitais e técnicas de coleta de dados no formato de perguntas e respostas previamente estruturadas. (Fontelles et al., 2011).

Foi elaborado um instrumento com 9 questões fechadas e de múltipla escolha, relacionadas ao tema de desempenho ocupacional, a partir do protocolo de avaliação COPM (Law et al., 2009). As informações colhidas dos participantes nesta etapa da pesquisa foram analisadas quantitativamente através da tabulação dos dados, por métodos estatísticos.

O estudo teve como área de abrangência o município de Belém, no Estado do Pará, na qual a unidade de análise foi o Centro de Ciências Biológicas e da Saúde - CCBS II, da Universidade do Estado do Pará (UEPA). A coleta ocorreu no período de 1 de junho a 30 de julho de 2021.

A mostra desta pesquisa foi composta por alunos dos cursos de graduação da área da saúde da Universidade do Estado do Pará, no município de Belém. Constituída por homens e mulheres, graduandos em um dos sete cursos oferecidos. Participaram do estudo 250 dos 660 indivíduos da população matriculados no ano de 2021, com a margem de erro de 5\% e com a taxa de confiabilidade de 95\% (Marotti, et al., 2008).

Foram critérios de inclusão deste estudo: homens e mulheres de todas as idades, matriculados em um dos sete cursos do Centro de Ciências Biológicas e da Saúde (CCBS); cursando entre o primeiro e último ano de seu curso; aceitar participar da pesquisa; concordar e assinar o Termo de Consentimento Livre Esclarecido - TCLE. Foram excluídos desse estudo, alunos de outros centros; alunos de outros campos fora da cidade de Belém e os que não tenham concordado com o TCLE.

Este estudo foi aprovado pelo Comitê de Ética em Pesquisa (CEP) com o registro 4.702 .912 e no dia 10 de maio de 2021. E está em consonância com a resolução 466/2012 do Conselho Nacional de Saúde (Brasil, 2013).

\section{Resultados e Discussões}

O contato inicial com os participantes foi realizado pelos grupos de aplicativo em redes sociais (Facebook, Instagram, Whatsapp), explicou-se sobre o objetivo da pesquisa e, posteriormente, foi feita a solicitação da autorização através da assinatura do Termo de Consentimento Livre e Esclarecido (TCLE) e o envio do formulário. Todos estes procedimentos foram realizados de maneira remota/virtual. A identidade dos participantes foi preservada.

Participaram da pesquisa 250 alunos (Gráfico 1), homens e mulheres, dos quais 72 estudantes cursavam Terapia Ocupacional, 60 estudantes Fisioterapia, 53 estudantes Medicina, 49 estudantes Biomedicina e 16 estudantes Saúde Coletiva. 
Gráfico 1: Relação das amostras por cursos de graduação.

\section{Relaçào das Amostras por Curso}

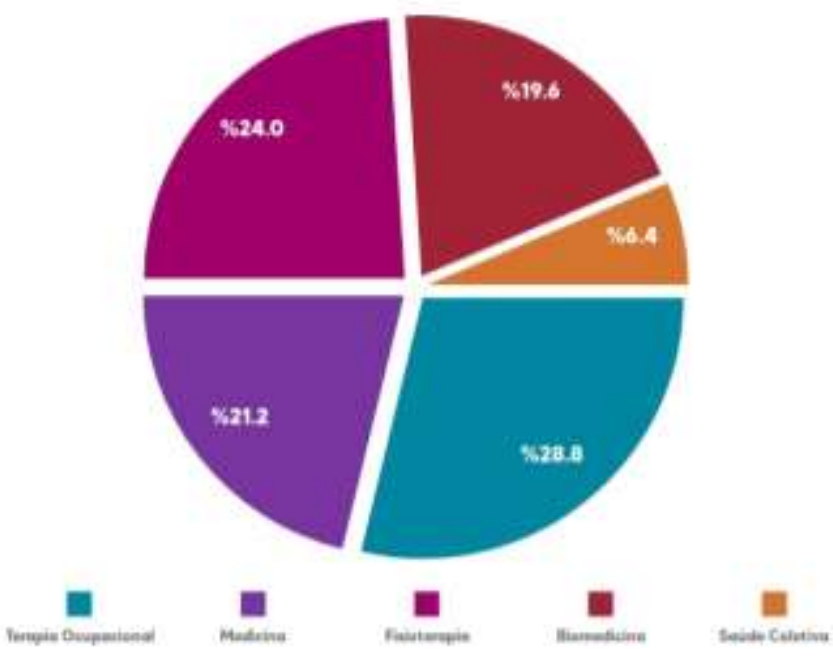

Fonte: Autores (2021).

Diante do cenário da pandemia da COVID-19, é considerável compreender a importância de se avaliar o desempenho ocupacional desses estudantes universitários, pois as áreas do autocuidado, produtividade e lazer possuem um impacto direto na qualidade de vida e em seus papéis sociais. Ademais, é também relevante considerar a importância, realização e satisfação dessas três esferas ocupacionais durante a pandemia (Pereira, 2021).

Durante a entrevista questionou-se a importância do autocuidado, produtividade e o lazer para os entrevistados a fim de verificar o grau de relevância atribuída a elas. Nesse viés, pode-se perceber que atribuir alto grau de relevância para essas atividades é de extrema seriedade, isso pois os componentes "autocuidado, produtividade e lazer" são as bases para a construção de uma rotina saudável, haja vista que alcança o equilíbrio entre produção, descanso e o bem estar (Caldas, 2011).

Dessa forma, as respostas para esse primeiro questionamento foram dispostas no quadro a seguir (Quadro 1). os participantes da pesquisa classificaram os componentes de autocuidado, produtividade e lazer como extremamente importantem importante e mediano.

Quadro 1: Relação das respostas acerca da importância do autocuidado, produtividade e o lazer.

\begin{tabular}{|l|c|c|c|}
\hline Importância & Autocuidado & Produtividade & Lazer \\
\hline Extremamente importante & $80 \%$ & $68 \%$ & $75 \%$ \\
\hline Importante & $20 \%$ & $30 \%$ & $20 \%$ \\
\hline Mediano & - & $2 \%$ & $5 \%$ \\
\hline Pouco importante & - & - & - \\
\hline Sem nenhuma importância & - & - & - \\
\hline
\end{tabular}

Fonte: Autores (2021).

Quando levantado o questionamento sobre a importância atribuída ao autocuidado no cotidiano, as amostras pontuaram: $80 \%$ disseram que o autocuidado é muito importante em seu cotidiano e $20 \%$ disseram que o autocuidado é importante. 
A prática do autocuidado se faz imprescindível na vida do ser humano, entretanto, devido ao enfrentamento da pandemia, esses cuidados precisam ser tomados de modo redobrado. Visto que o estresse, a preocupação, a ansiedade e sentimentos depressivos se acentuaram seriamente durante a quarentena.

Portanto, a necessidade de momentos de autocuidado é essencial para que se mantenha ou otimize a saúde mental e física durante o isolamento social (Santana, 2020).

Ao serem interrogados sobre qual a importância que é atribuída a produtividade no cotidiano, observou-se que $68 \%$ dos alunos disseram que a produtividade é muito importante; $30 \%$ dos alunos disseram que a produtividade é importante e $2 \%$ afirmaram que a produtividade é parcialmente importante.

De acordo com Gusy (2021), entre a população estudantil mundial, a produtividade possui alto grau de relevância pois, devido as altas demandas da faculdade e inúmeras exigências do mercado de trabalho, o aluno se sente imposto a estudar copiosamente todos os dias, a fim de corresponder expectativas frequentemente extravagantes. Tal afirmação pode ser corroborada a partir dos resultados obtidos, os quais demonstram que a grande maioria dos alunos conferem muito valor à produtividade.

Quando questionados “Qual a importância que é atribuída ao Lazer no cotidiano?”, as amostras pontuaram que 75\% dos estudantes acreditam que o lazer é extremamente importante; $2 \%$ pontuaram que o lazer é importante e $5 \%$ disseram que a importância do lazer é mediana.

O lazer é uma prática social que faz parte do cotidiano de cada ser humano, e precisa ser entendida como necessidade básica na vida.

Dessa maneira, é imprescindível que haja tempo e um espaço social adequado para a prática do lazer. Além disso, é notável como se torna mais imprescindível em sociedades industrializadas, devido ao alto nível de estresse perpetuado (Gomes, 2014).

Dentro do campo da importância atribuída as atividades de autocuidado, produtividade e lazer, vale ressaltar a realização dessas atividades diante do cenário pandêmico. O engajamento dos estudantes universitários na realização de tais atividades incluíram atividades de autocuidado, atividades acadêmicas curriculares e extracurriculares na modalidade de ensino a distância; atividades de lazer, passatempos, atividades recreativas, e etc.

Diante disso, identificou-se que o grau de realização dessas atividades se mostrou reduzido durante o período de isolamento social, conforme observado no quadro abaixo (Quadro 2). Isso se dá pois, quando o ser humano, que é considerado um ser social, não está circundado de outras pessoas, o nível de engajamento nas atividades básicas do dia-a-dia tende a diminuir. Somado a isso, observa-se, ainda, o grande sofrimento psicológico causado pelo momento histórico da pandemia. Por conseguinte, a realização satisfatória desses afazeres se torna grandemente dificultosa. (Medeiros et al., 2020). O quadro a seguir exibe a realização das atividades de autocuidado, produtividade e lazer por parte dos estudantes.

Quadro 2: Relação das respostas acerca da realização das atividades do autocuidado, produtividade e o lazer.

\begin{tabular}{|l|c|c|c|}
\hline Realização & Autocuidado & Produtividade & Lazer \\
\hline Realizou bem & $9 \%$ & $4 \%$ & $5 \%$ \\
\hline $\begin{array}{l}\text { Realizou, porém não como } \\
\text { esperado }\end{array}$ & $26 \%$ & $15 \%$ & $21 \%$ \\
\hline Realizou pela metade & $12 \%$ & $10 \%$ & $45 \%$ \\
\hline $\begin{array}{l}\text { Tentou mas não conseguiu } \\
\text { como esperado }\end{array}$ & $32 \%$ & $35 \%$ & $26 \%$ \\
\hline Não conseguiu realizar & $21 \%$ & $36 \%$ & \\
\hline
\end{tabular}

Fonte: Autores (2021). 
Ao serem indagados "Você conseguiu realizar atividades de autocuidado no período de quarentena e isolamento social?", constatou-se que $21 \%$ dos estudantes não conseguiram realizá-las de nenhuma forma; $32 \%$ dos estudantes tentaram, mas não conseguiram realizá-las; $12 \%$ conseguiram realizá-las parcialmente; $26 \%$ tentaram e conseguiram realizá-las; $9 \%$ conseguiam realizá-las sem quaisquer intercorrências.

Quando perguntados "Você conseguiu realizar atividades produtivas em casa no período de quarentena e isolamento social?", arrecadou-se as seguintes respostas: $36 \%$ não conseguiram realizá-las de nenhuma forma; 35\% tentaram, mas não conseguiram realizá-las; $10 \%$ conseguiram realizá-las parcialmente; $15 \%$ tentaram e conseguiram realizá-las; $4 \%$ conseguiram realizá-las sem quaisquer intercorrências.

Ao serem perguntados se conseguiam realizar atividades de lazer em casa, em meio a quarentena e isolamento social, pôdese observar que $26 \%$ dos alunos não conseguiram realizá-las de nenhuma forma; $45 \%$ tentaram desempenhar as atividades, mas não conseguiram; $21 \%$ conseguiram realizá-las parcialmente; $5 \%$ tentaram e conseguiram realizá-las e 3\% afirmam que conseguiram realizar suas atividades de lazer sem quaisquer intercorrências.

Santana (2020) afirma que o autocuidado é a prática de cuidados realizados pelo sujeito para preservar a sua vida, saúde e bem-estar, sendo um elemento essencial para o desenvolvimento do ser. Essa forma de promoção à saúde é uma maneira de demonstrar afeto e respeito para com seu Eu físico e mental, buscando sanar todas as necessidades que o corpo e a mente exigem.

Tendo em vista a importância do autocuidado para formação do indivíduo, é possível afirmar que sem a prática dessa ação básica, ocorre a diminuição da produtividade e interesse em quaisquer outras atividades da vida do indivíduo como o lazer. Tal pensamento é corroborado com os dados mostrados acima onde a maioria das pessoas não conseguiram realizar plenamente o autocuidado e, consequentemente, também não conseguiram realizar completamente as atividades produtivas e de lazer.

Diante dos dados do quadro anterior, o grau de satisfação das atividades avaliadas foi proposto com o intuito de reforçar os dados mensurados, uma vez que $86 \%$ dos alunos pontuam que não realizaram as atividades como esperado ou como planejado. Assim, esses dados refletem diretamente no grau de satisfação em realizá-las em casa durante o período de pandemia, os resultados apontaram que no que se refere ao autocuidado, produtividade e lazer a maioria dos participantes relataram que sentiam-se nada satisfeitos ou pouco satisfeitos, confome identifica-se no quadro a seguir (Quadro 3).

Quadro 3: relação das respostas acerca da satisfação com o autocuidado, produtividade e o lazer.

\begin{tabular}{|l|c|c|c|}
\hline Satisfação & Autocuidado & Produtividade & Lazer \\
\hline Extremamente satisfeito & $8 \%$ & $0 \%$ & $2 \%$ \\
\hline Satisfeito & $15 \%$ & $10 \%$ & $4 \%$ \\
\hline Mediano & $10 \%$ & $32 \%$ & $24 \%$ \\
\hline Pouco satisfeito & $35 \%$ & $31 \%$ & $32 \%$ \\
\hline Nada satisfeito & $32 \%$ & $27 \%$ & $38 \%$ \\
\hline
\end{tabular}

Fonte: Autores (2021).

Quando levantado o questionamento sobre o nível de satisfação no que se refere à prática de atividades de autocuidado em casa, averiguou-se: $32 \%$ dos participantes estão insatisfeitos por não realizarem atividades de autocuidado de maneira nenhuma; $35 \%$ tentaram, mas ficaram insatisfeitos por não conseguirem realizá-las; $10 \%$ ficaram parcialmente satisfeitos com a realização do autocuidado em casa; $15 \%$ ficaram satisfeitos por realizarem o autocuidado em casa; $8 \%$ ficaram extremamente satisfeitos por realizarem atividades de autocuidado em casa.

Segundo Gomes (2014), quando uma necessidade humana não é satisfatória de maneira adequada gera uma pobreza na vivência do indivíduo, seja de felicidade, afeto, entendimento, capacidade cognitiva, autoestima, entre outros. Então, pôde-se 
Research, Society and Development, v. 11, n. 1, e49211125229, 2022

(CC BY 4.0) | ISSN 2525-3409 | DOI: http://dx.doi.org/10.33448/rsd-v11i1.25229

perceber diante dos altos níveis de insatisfação que a falta de lazer, produtividade e autocuidado afetou de forma grandiosa no contentamento da maioria dos estudantes.

Ao serem questionados sobre o nível de satisfação no que se refere ao desempenho de atividades produtivas em casa, responderam: $27 \%$ estão insatisfeitos por não realizarem atividades produtivas de maneira nenhuma; $31 \%$ tentaram, mas ficaram insatisfeitos por não conseguirem desempenhá-las; $32 \%$ ficaram parcialmente satisfeitos com a realização em casa; $10 \%$ ficaram satisfeitos em realizar em casa.

Como pode ser observado, é alta a percentagem de alunos que se sentiram insatisfeitos em relação ao nível de produtividade durante a quarentena. Tal fato é justificado devido à banalização do ato de trabalhar/estudar quando se está doente ou com altos níveis de estresse, a ponto de diminuir a eficiência da atividade. É extremamente comum se deparar com pessoas fisicamente incapacitadas de trabalhar e/ou alunos mentalmente exaustos para estudar, mas que, ainda assim, frequentam de maneira regular seu local de ocupação (Gusy, 2021).

É importante frisar que, por vezes, essa atitude é considerada louvável por muitos pais, colegas de trabalho e, até mesmo, pelo próprio indivíduo. Sendo assim, o contexto de pandemia aflorou em estudantes a necessidade de serem produtivos mesmo que a sua saúde mental, e por vezes física, esteja comprometida. Afinal, têm-se a falsa e errônea ideia de que, por se estar em casa, o estudo e o trabalho se tornam mais fáceis de serem realizados, logo, se tornam obrigatórios.

Quando levantado o questionamento sobre o nível de satisfação com a prática das atividades de lazer, obtiveram-se as seguintes respostas: $38 \%$ estão insatisfeitos por não realizarem atividades de lazer de maneira nenhuma; 32\% tentaram, mas ficaram insatisfeitos por não conseguirem realizá-las; $24 \%$ ficaram parcialmente satisfeitos com o desempenho do lazer em casa; $4 \%$ ficaram satisfeitos ao realizarem o lazer em casa, $2 \%$ ficaram extremamente satisfeitos ao desempenhar atividades de lazer em casa.

Acácio et al. (2021) pontua em sua pesquisa a importância significativa das atividades de lazer na vida dos estudantes universitários de Terapia Ocupacional da Universidade Estadual de Ciências da Saúde de Alagoas, pois estes promovem uma melhor qualidade de vida e um bom desempenho nas atividades acadêmicas e extracurriculares. $\mathrm{O}$ autor conclui se é necessário pesquisar o desempenho ocupacional no lazer durante o período da pandemia de COVID-19.

O cenário da pandemia trouxe para esses estudantes em sua maioria muitas implicações em seu desempenho ocupacional, já que a razão entre a importância atribuída as atividades de autocuidado, produtividade e lazer e as suas realizações, desde o resultado esperado não satisfatório até o não consegui realizá-las, mostra que 9 entre 10 amostras não realizaram suas ocupações como esperado durante o período da pandemia. Esses dados são reforçados com a baixa taxa de satisfação serem em média de 1,83\% do total das respostas.

O estudo de Teixeira e Dahl (2020) aponta um conjunto de elementos e estratégias fundamentais para promoção da saúde mental e apoio psicossocial da comunidade universitária, sobretudo dos estudantes. As ações mostram que é possível promover estratégias de melhora no desempenho ocupacional e que podem ir além desse foco, podendo se expandir para a esfera psicossocial, como estratégias de atividade grupal, práticas complementares e integrativas, adaptação a modalidade remota e outras atividades educativas complementares de autocuidado, produtividade e lazer

Assim, observa-se que de maneira geral a pandemia de COVID 19 gerou impactos negativos nos mais variados âmbitos da vida dos estudantes e que diante desse contexto, muitos estudantes se veem na obrigação de ser produtivo e de realizar suas rotinas e atividades de maneira plena mesmo que haja diversos fatores interferindo nesse processo, o que gera mais sofrimento psicológico e emocional. 
Research, Society and Development, v. 11, n. 1, e49211125229, 2022

(CC BY 4.0) | ISSN 2525-3409 | DOI: http://dx.doi.org/10.33448/rsd-v11i1.25229

\section{Conclusão}

Diante do cenário pandêmico, foi observado que o súbito afastamento social influenciou negativamente no desempenho ocupacional de estudantes da Universidade do Estado do Pará, no que diz respeito a atividades de autocuidado, produtividade e lazer. Observou-se que os estudantes, em sua maioria, atribuíam alto grau de importância para tais práticas. Entretanto, eles experienciaram grande dificuldade de realizar essas rotinas e tarefas, o que resultou em grandes níveis de frustração pelo não cumprimento. Destacase que a prática do autocuidado é fundamental para que a saúde física e mental seja mantida, e como a falta da realização satisfatória dessa atividade, os malefícios ocasionados no âmbito psicológico e emocional do indivíduo é iminente. Somado a isso, há ainda as repercussões negativas da insuficiência de atividades produtivas, haja vista que os alunos implicam altos níveis de relevância às atividades produtivas.

Portanto, corrobora-se que a presente pesquisa reconhece a grande relevância de observar e medir os impactos do isolamento social na saúde mental de estudantes universitários. A fim de que, à posteriori, a partir da contribuição para a literatura sobre o assunto, sejam criadas medidas para a diminuição de danos psicológicos dos alunos causados pela pandemia.

Por fim, em pesquisas futuras, sugere-se a exploração de campos da saúde biopsicossocial que possam ser acometidos com o distanciamento social e com os impactos psicológicos da pandemia no cotidiano ocupacional em diferentes públicos, em especial, profissionais da saúde.

\section{Referências}

Acácio, M. da S., Reis, M. C. da S., Moreira, S. L. de B., Lins, A. E. dos S., Souza, M. A., \& Barros, A. R. (2021). Leisure: a significant occupation in occupational therapy students. Research, Society and Development, 10(11), e89101119442. https://doi.org/10.33448/rsd-v10i11.19442

Brasil. (2013) Resolução 466/12 do Conselho Nacional de Saúde Sobre Diretrizes e Normas Regulamentadoras de Pesquisa envolvendo seres humanos. Brasília (DF) http://www.uniararas.br/documentos/DOC00012.pdf.

Brasil. (2020). Lei n ${ }^{\circ}$ 13.979, de 6 de fevereiro de 2020. Medidas para enfrentamento da emergência de saúde pública de importância internacional decorrente do coronavírus responsável pelo surto de 2019. Brasília-DF. http://www.planalto.gov.br/ccivil_03/_ato2019-2022/2020/lei/113979.htm.

Brooks, S. K., Webster, R. K., Smith, L. E., Woodland, L., Wessely, S., Greenburg, N., Rubin, G. J. (2020). The Psychological Impact of Quarantine and How to Reduce It: Rapid Review of the Evidence. The Lancet; 395:912-920.

Caldas, A. A. C. Facundes, V. L. D., Silva, H. J. (2011). O uso da medida canadense de desempenho ocupacional em estudos brasileiros: uma revisão sistemática. Rev. ter. ocup., 22(3): 238-244.

Costa, E., Oliveira, L., Corrêa, V., \& Folha, O. (2017). Ciência Ocupacional e Terapia Ocupacional: algumas reflexões/ Occupational Science and Occupational Therapy: some reflections. Revista Interinstitucional Brasileira de Terapia Ocupacional - REVISBRATO, 1(5), 650-663.

De Troi, M., \& Quintilio, W. (2020.). Coronavírus: Lições anti-negacionistas e o futuro do planeta. SciELO em Perspectiva. https://blog.scielo.org/blog/2020/03/31/coronavirus-licoes-anti-negacionistas-e-o-futuro-do-planeta/

Ferrari, A; Cunha, A.M. (2020) A pandemia de Covid-19 e o isolamento social: Saúde versus economia - Coronavírus. (n.d.). UFRGS | Universidade Federal do Rio Grande do Sul. https://www.ufrgs.br/coronavirus/base/artigo-a-pandemia-de-covid-19-e-o-isolamento-social-saude-versus-economia/.

Gusy, B., Lesener, T., \& Wolter, C. (2021). Time Pressure and Health-Related Loss of Productivity in University Students: The Mediating Role of Exhaustion. Frontiers in public health, 7;9:653440

Gomes, C. L. (2014). Lazer: necessidade humana e dimensão da cultura. Revista Brasileira de Estudos do Lazer, 1(1), 13-20.

Law, M.; Baptiste, S.; Carswell, A.; Mccoll, M. A.; Polatajko, H. L.; Pollock, N. (2009). Medida Canadense de Desempenho Ocupacional (COPM). Trad. Lívia de Castro Magalhães, Lilian Vieira Magalhães e Ana Amélia Cardoso. Belo Horizonte: Editora Universidade Federal de Minas Gerais.

Marotti, J., Mantelli, A; Furuyama, R., Pigozzo, M., Campos, T., \& Laganá, D. C (2008). Amostragem em pesquisa clínica: Tamanho da amostra. Ver odontol Unicid. 20(2):186-194.

Medeiros, A. Y. B. B. V. de, Pereira, E. R., Silva, R. M. C. R. A., \& Dias, F. A. (2020). Psychological phases and meaning of life in times of social isolation due the COVID-19 pandemic a reflection in the light of Viktor Frankl. Research, Society and Development, 9(5), e122953331. https://doi.org/10.33448/rsd-v9i5.3331

Mota, J. d. S. (2019). Utilização do google forms na pesquisa acadêmica. Revista Humanidades e Inovação, 6(12), 371-380. 
Research, Society and Development, v. 11, n. 1, e49211125229, 2022

(CC BY 4.0) | ISSN 2525-3409 | DOI: http://dx.doi.org/10.33448/rsd-v11i1.25229

Pereira, G. C. (2021). Impacto da pandemia da COVID-19 sobre o perfil de desempenho ocupacional de pessoas idosas participantes da oficina Deu Branco da Universidade Federal de São Paulo no campus Baixada Santista. [Trabalho de Conclusão De Curso]. Universidade Federal De São Paulo.

M. E. D. (2020). O autocuidado diante de uma pandemia mundial. [Trabalho de conclusão de curso]. Faculdade Pernambucana de Saúde. 01-20.

Uepa. (2020). Governo do Pará suspende aulas na rede estadual. Belém-PA. https://www.uepa.br/pt-br/noticias/governo-do-par\%C3\%A1-suspende-aulas-na-redeestadual. 